\title{
Characterization and identification of newly isolated Acinetobacter baumannii strain Serdang 1 for phenol removal
}

\begin{abstract}
A new indigenous bacterial strain from Malaysian soil contaminated with petroleum waste had been successfully isolated, characterized and identified for phenol removal. The gram negative bacteria showed $98 \%$ identity with Acinetobacter baumannii based on BiologE Identification System and the determination of a partial 16S ribosomal RNA (rRNA) sequence. The isolate clustered with species belonging to Acinetobacter clade in a $16 \mathrm{~S}$ rDNA-based neighbour-joining phylogenetic tree.
\end{abstract}

Keyword: 16S ribosomal RNA; Acinetobacter baumannii; Phenol; Phylogenetic tree analysis 